\title{
Novel Compound Heterozygous Mutations in the Cathepsin K Gene in Japanese Female Siblings with Pyknodysostosis
}

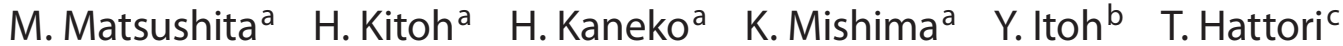 \\ N. Ishiguro ${ }^{a}$
}

Departments of a Orthopaedic Surgery and ${ }^{b}$ Medical Research Engineering, Nagoya University School of Medicine, Nagoya, and 'Department of Orthopaedic Surgery, Aichi Children's Health and Medical Center, Obu, Japan

\section{Key Words}

Cathepsin K · Chondroitin 4-sulfate $\cdot$ Compound

heterozygous mutations $\cdot$ Pyknodysostosis

\begin{abstract}
We report on female siblings with pyknodysostosis who showed common clinical and radiographic features including disproportionate short stature, dental abnormalities, increased bone density, open fontanelle, and acroosteolysis. Sequence analysis of the cathepsin K (CTSK) gene demonstrated compound heterozygous mutations (935 C>T, A277V and 489 G > C, R122P) in the affected siblings and a heterozygous mutation in their parents. The former missense mutation has previously been reported in 6 unrelated patients, and the latter seemed to be a novel mutation. Atomic model assessment of the CTSK gene revealed that the R122P mutant could disrupt hydrogen bonds binding with chondroitin 4-sulfate leading to a decrease in the collagen-degrading activity of cathepsin $\mathrm{K}$.

Copyright $\odot 2012$ S. Karger AG, Basel
\end{abstract}

Pyknodysostosis (MIM 265800) is an uncommon autosomal recessive skeletal dysplasia characterized by osteosclerosis, short stature, acroosteolysis of the distal phalanges, bone fragility, hypoplastic clavicle, and skull deformities with delayed suture closure [Sedano et al., 1968; Kawahara et al., 1977; Edelson et al., 1992]. It is caused by mutations in the cathepsin K (CTSK) gene [Hou et al., 1999], a lysosomal cysteine protease that is highly expressed exclusively in osteoclasts and critical for bone remodeling and resorption by osteoclasts [Tezuka et al., 1994]. The CTSK gene spans approximately $12 \mathrm{~kb}$ on 1q21 and contains 8 exons. Thirty-three different mutations, spread throughout the whole gene, have been reported to date in patients with pyknodysostosis [Michela et al., 2007; Schilling et al., 2007; Li et al., 2009; Naeem et al., 2009; Khan et al., 2010; Toral-López et al., 2011].

In the present study, we describe the clinical and radiographic findings of female siblings of Japanese origin with pyknodysostosis and performed molecular analysis of the CTSK gene in their family.

\section{Case Reports}

Two female siblings, an 11-year-old elder sister and an 8-yearold younger sister, were first seen at our hospital because of short stature when they were 3 years and 1 year and 3 months old, respectively. They are the first and the second child of young, healthy, nonconsanguineous parents. The height of the elder sister and the younger sister was $118.8 \mathrm{~cm}(-3.97 \mathrm{SD})$ and $109.9 \mathrm{~cm}$

\section{KARGER}

Fax +4161306 1234

E-Mail karger@karger.ch

www.karger.com
(C) 2012 S. Karger AG, Basel

$1661-8769 / 11 / 0026-0254 \$ 38.00 / 0$

Accessible online at:

www.karger.com/msy
Dr. Hiroshi Kitoh

Department of Orthopaedic Surgery, Nagoya University School of Medicine 65 Tsurumai-cho, Showa-ku

Nagoya, Aichi 466-8550 (Japan)

Tel. +81 52741 2111, E-Mail hkitoh@med.nagoya-u.ac.jp 


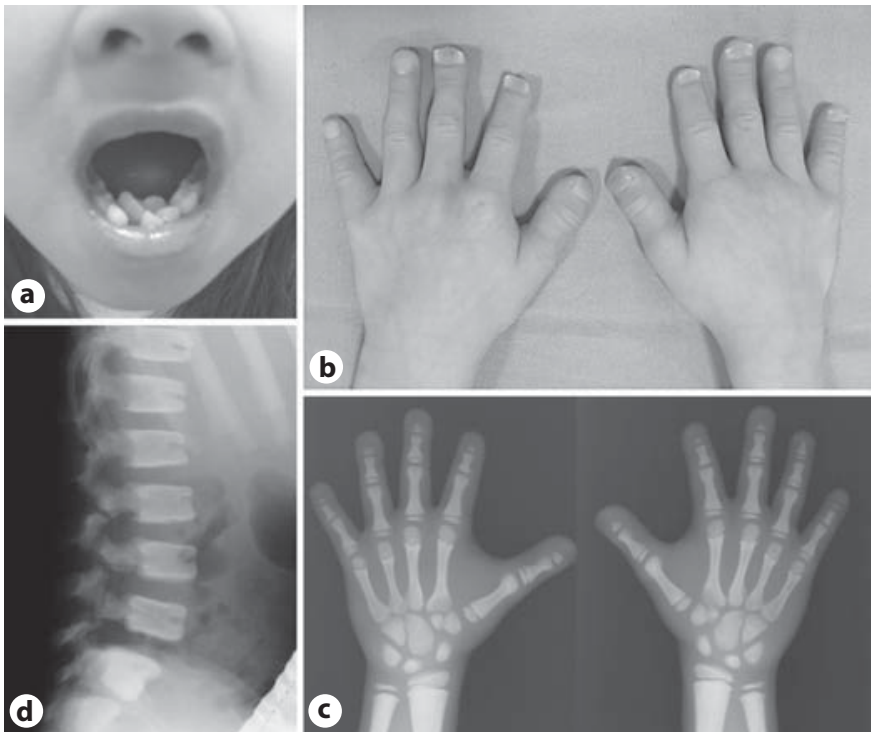

Fig. 1. Clinical photographs of the elder sister at the age of 11 years demonstrating marked irregular position of the permanent teeth (a), and short fingers with loose digital skin folds and flattened nails (b). Anteroposterior hand and lateral lumbar spine radiographs of the younger sister at the age of 8 years showing generalized osteosclerosis, shortening of distal phalanges (acroosteolysis) (c), and mild spondylolysis of the third, fourth and fifth lumbar vertebrae $(\mathbf{d})$.

$(-3.35$ SD), respectively. Their arm spans were 107.7 and $103.6 \mathrm{~cm}$, respectively. The elder sister had a history of 2 tibial fractures while the younger sister had had 2 tibial fractures, one metatarsal fracture and one acromial fracture. Both showed similar clinical manifestations, including disproportionate short-limbed dwarfism, prominent calvaria with small facial structures, obtuse mandibular angle, narrow grooved palate, dental abnormalities, stubby hands with loose digital skin folds and flattened nails (fig. 1a, b). Increased bone density, opened anterior fontanelle with separated cranial sutures and partial aplasia of distal phalanges (acroosteolysis) were seen in both patients radiographically (fig. 1c). In addition, spondylolysis of the third, fourth and fifth lumbar vertebrae were observed in the younger sister (fig. 1d). Laboratory tests including complete blood counts, calcium and phosphorous levels, liver and kidney functions, insulin-like growth factor-1, and insulin-like growth factor binding protein-3 were all within normal values. On the basis of characteristic clinical and radiographic features, the diagnosis of pyknodysostosis was verified.

\section{Molecular Analysis of the CTSK Gene}

After informed consent was obtained from all family members, genomic DNA was extracted from peripheral blood leukocytes of the 2 patients and their normal par- ents using the QIAamp DNA Blood Midi kit (QIAGEN Inc., Valencia, Calif., USA). The exons (2-8) and their flanking intronic regions of the CTSK gene were amplified by PCR using sets of primers designed on the genomic sequence (table 1). The mutation analysis of the complete coding sequence of the CTSK gene and exon-intron boundaries [Gelb et al., 1997] was performed using the CEQ 8000 Sequencer (Beckman Coulter, Fullerton, Calif., USA) according to the manufacturer's instructions. Direct sequence analysis of the patients' genomic DNA demonstrated the heterozygous presence of the missense mutations g.489 G $>$ C (p.Arg122Pro) in exon 4 and g.935 C $>$ T (p.Ala277Val) in exon 7 of the CTSK gene (fig. 2). Analysis of their parents revealed the R122P mutation was derived from the mother and the A277V mutation from the father. The latter mutation was reported previously in some patients with pyknodysostosis [Gelb et al., 1998; Hou et al., 1999; Fujita et al., 2000; Donnarumma et al., 2007], but the former seemed to be a novel mutation.

\section{In Silico Analysis}

The potential causal effect of the novel missense mutation (R122P) was predicted by using different software: PolyPhen-2 (http://genetics.bwh.harvard.edu/pph2/) [Adzhubei et al., 2010], PMut (http://mmb2.pcb.ub.es:8080/ PMut/) [Ferrer-Costa et al., 2005] and SIFT (http://sift. jcvi.org/) [Kumar et al., 2009]. The R122P missense mutation in CTSK was predicted to have a pathogenic effect by PolyPhen-2, PMut and SIFT software, which showed 'probably damaging', 'pathological' and 'affect protein function', respectively. We also examined evolutionary conservation of the mutated residue and surrounding amino acids. This analysis demonstrated that arginine at position 122 in CTSK is a highly conserved amino acid in different species and human cathepsin subfamilies (fig. 3).

\section{Cathepsin K Atomic Model Assessment}

Li et al. [2002] demonstrated that the unique triplehelical collagen-degrading activity of cathepsin $\mathrm{K}$ depends on the formation of complexes with chondroitin 4-sulfate (C4-S). The 3-dimensional protein structure of cathepsin $\mathrm{K}$ and C4-S was available from Protein Databank (PDB code: 3C9E) [Li et al., 2008]. The mutant model was built by Rotamer Explorer of MOE (Molecular Operating Environment, Chemical Computing Group Inc., Canada) according to the manufacture's instructions. 
Fig. 2. Direct sequencing of the CTSK gene in the affected elder sister, her father and her mother. The data of the affected younger sister, which are the same as those of the elder sister, are not shown. A novel heterozygous missense mutation in exon 4 predicting an amino acid change from arginine to proline at position 122 was demonstrated in the elder sister and her mother. The heterozygous missense mutation in exon 7 predicting an amino acid change from alanine to valine at position 277 was observed in the elder sister and her father.

Fig. 3. In silico analysis of the R122P missense mutation in the CTSK gene predicting probably damaging by PolyPhen-2, pathological by PMut and affect protein function by SIFT software [Ferrer-Costa et al., 2005; Kumar et al., 2009; Adzhubei et al., 2010]. Conservation analysis shows a highly conserved arginine at position 122 in the CTSK gene in different species and human cathepsin subfamilies.

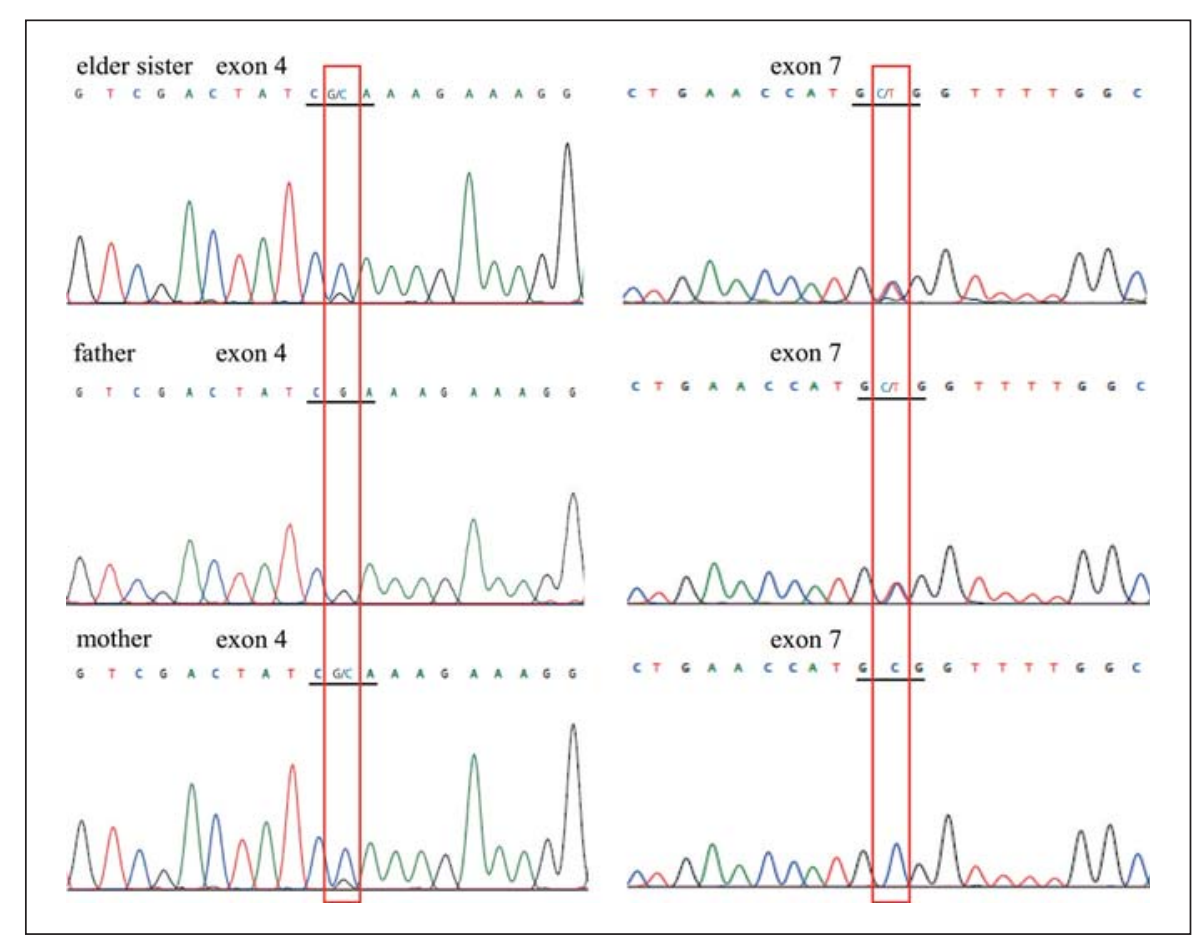

Then, the molecular docking simulation was carried out by MOE/Dock. The force field used in the minimizations presented here was the Merck Molecular Force Field 94x (MMFF94x), which is suitable for ligand and protein [Halgren, 1996]. The final conformations were selected by comparing calculated Gibbs free energy of candidates. The interaction energies of C4-S to cathepsin K were calculated as follows:

$$
\Delta \mathrm{E}=\mathrm{E}_{\text {complex }}-\left(\mathrm{E}_{\text {cathepsin } \mathrm{K}}+\mathrm{E}_{\mathrm{C} 4-\mathrm{S}}\right)
$$




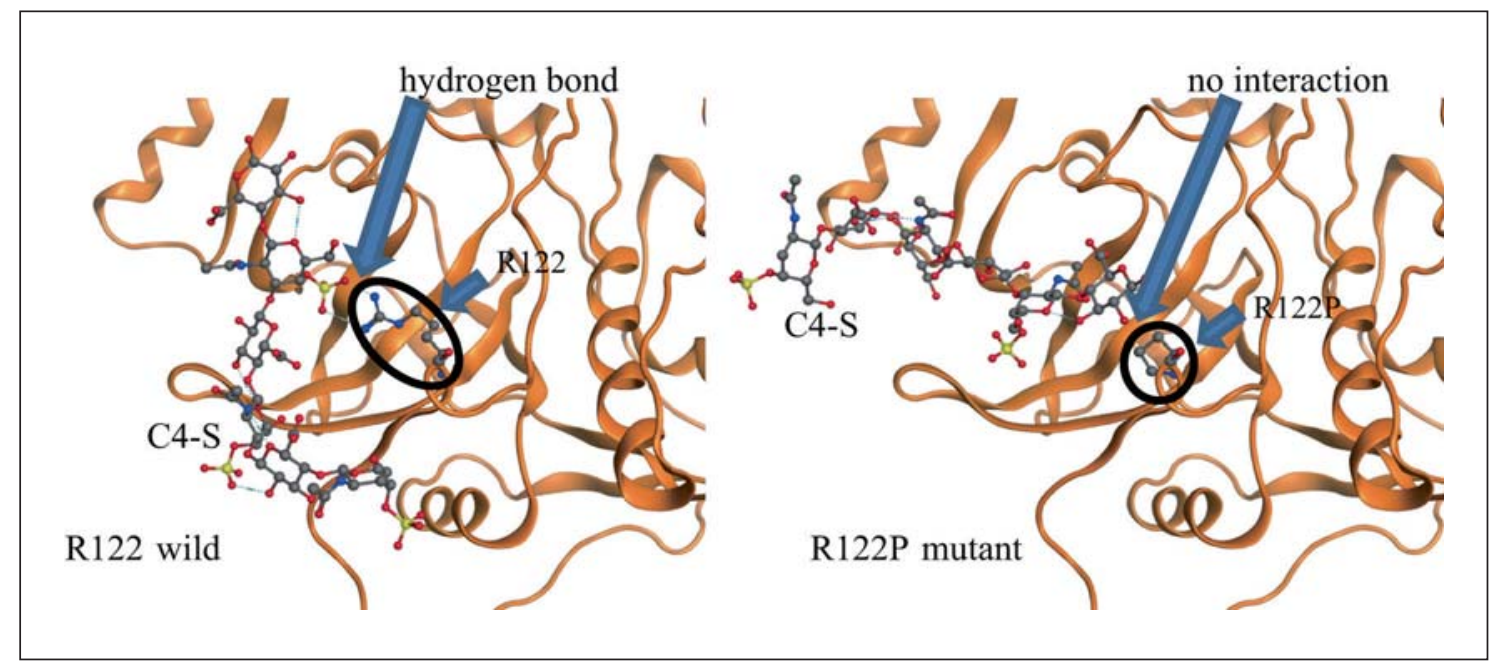

Fig. 4. The interaction energies of C4-S to the wild type and the R122P mutant of cathepsin K were -93.486 and $24.282 \mathrm{kcal}$, respectively. In the wild type of cathepsin K, R122 interacts directly via hydrogen-bonded ion pairs with C4-S. However, R122P disrupts hydrogen bonds binding with C4-S.

Table 1. List of primers used for PCR-amplification of the CTSK gene

\begin{tabular}{lll}
\hline Exon & Forward $\left(5^{\prime} \rightarrow 3^{\prime}\right)$ & Reverse $\left(5^{\prime} \rightarrow 3^{\prime}\right)$ \\
\hline 2 & TGG AAC CAG ATG TAC CAG CA & GGG AAG AGG GAC TGA TTT GC \\
3 & TCC ATG GTT AGT TCC CCA AC & GGG CTC TAC CTT CCC ATT CT \\
4 & CCT TGA GGC TTC TCT TGG TG & AAT TCC TTG CCC TGT TTT CC \\
5 & GCG GAA GAG GAA GAC TCA CA & AAA ACA TCA TGC TGG GGA AG \\
6 & ACC CTA GAC TGC TGC CTC TG & GGC CAC CTC CAT GTG AAT AC \\
7 & CTG CAG TAT GGA GCA GCA TC & TGG CAA TGC AAA ATG GAC TA \\
8 & TGC CTT CTC CCC TAT TTC TTG A & TGG GTG GAG AGA AGC AAA GT \\
\hline
\end{tabular}

$\mathrm{E}_{\text {cathepsin K }}, \mathrm{E}_{\mathrm{C} 4-\mathrm{S}}$ and $\mathrm{E}_{\text {complex }}$ are the energies of cathepsin K, C4-S and the complex of cathepsin K and C4$\mathrm{S}$, respectively. The interaction energies of C4-S to the wild type and the R122P mutant of cathepsin $\mathrm{K}$ were -93.486 and $24.282 \mathrm{kcal}$, respectively. In the wild type of cathepsin $\mathrm{K}$, the R122 interacts directly via hydrogenbonded ion pairs with C4-S. However, the R122P could disrupt hydrogen bonds binding with C4-S (fig. 4).

\section{Discussion}

We report on female siblings with autosomal recessive pyknodysostosis from a nonconsanguineous family of Japanese descent, who showed compound heterozygous mutations (935 C > T, A277V and $489 \mathrm{G}>\mathrm{C}, \mathrm{R} 122 \mathrm{P}$ ) in the
CTSK gene. The A277V mutation has previously been reported in 6 unrelated patients, one of whom is of Japanese origin [Gelb et al., 1998; Hou et al., 1999; Fujita et al., 2000; Donnarumma et al., 2007]. The A277 residue is not only highly conserved among the papain family members, but also immediately next to the invariably conserved $\mathrm{H} 276$ residue that forms the ion pair with the active cysteine (C139) to affect protein catalysis [Berti and Storer, 1995]. Expression studies with several missense mutants affecting the mature region of the CTSK gene showed that the A277V mutant had no detectable mature protein or enzyme activity [Hou et al., 1999]. It was anticipated that the A277V mutation resulted in a precursor protein lacking auto-activating function and unstable in the presence of pepsin. 
Li et al. [2002] demonstrated that the unique triplehelical collagen-degrading activity of cathepsin $\mathrm{K}$ depends on the formation of complexes with bone or cartilage resident glycosaminoglycans, such as C4-S. The stabilizing interactions between cathepsin $\mathrm{K}$ and C4-S are predominantly hydrogen bonding and ion pairs. Four basic residues (R122, K123, K124, and K305) interact directly via hydrogen-bonded ion pairs with C4-S. Especially, a basic amino acid triplet (R122, K123, K124), which is highly conserved among the several cathepsin subfamilies, is the most important set of interactions and forms multiple hydrogen bonds to C4-S. Moreover, the interaction between the R122 and C4-S seemed to be the strongest [Li et al., 2008]. The atomic model assessment demonstrated that the R122P mutant disrupts hydrogen bonds binding with C4-S and affects stabilizing interaction between cathepsin $\mathrm{K}$ and $\mathrm{C} 4-\mathrm{S}$. In silico analysis also revealed that the $\mathrm{R} 122 \mathrm{P}$ missense mutation in CTSK has a functional effect. The R122P mutation may affect proteolytic activity of cathepsin $\mathrm{K}$ by disrupting hydrogen bonds binding with chondroitin sulfate.

\section{References}

-Adzhubei IA, Schmidt S, Peshkin L, Ramensky VE, Gerasimova A, et al: A method and server for predicting damaging missense mutations. Nat Methods 7:248-249 (2010).

Berti PJ, Storer AC: Alignment/phylogeny of the papain superfamily of cysteine proteases. J Mol Biol 246:273-283 (1995).

-Donnarumma M, Regis S, Tappino B, Rosano C, Assereto S, et al: Molecular analysis and characterization of nine novel CTSK mutations in twelve patients affected by pycnodysostosis. Mutation in brief \#961. Online. Hum Mutation 28:524-533 (2007).

Edelson JG, Obad S, Geiger R, On A, Artul HJ: Pycnodysostosis. Orthopedic aspects with a description of 14 new cases. Clin Orthop Relat Res 280:263-276 (1992).

-Ferrer-Costa C, Gelpí JL, Zamakola L, Parraga I, de la Cruz X, Orozco M: PMUT: a web-based tool for the annotation of pathological mutations on proteins. Bioinformatics 21:31763178 (2005).

- Fujita Y, Nakata K, Yasui N, Matsui Y, Kataoka E, et al: Novel mutations of the cathepsin K gene in patients with pycnodysostosis and their characterization. J Clin Endocrinol Metab 85:425-431 (2000).

Gelb BD, Shi GP, Heller M, Weremowicz S, Morton C, et al: Structure and chromosomal assignment of the human cathepsin K gene. Genomics 41:258-262 (1997).
Gelb BD, Willner JP, Dunn TM, Nataline B, Kardon NB, et al: Paternal uniparental disomy for chromosome 1 revealed by molecular analysis of a patient with pycnodysostosis. Am J Hum Genet 62:848-854 (1998).

Halgren TA: Merck Molecular Force Field. II. MMFF94 van der Waals and Electrostatic Parameters for Intermolecular Interactions. J Comput Chem 17:520-552 (1996).

Hou WS, Brömme D, Zhao Y, Mehler E, Dushey $\mathrm{C}$, et al: Characterization of novel cathepsin $\mathrm{K}$ mutations in the pro and mature polypeptide regions causing pycnodysostosis. J Clin Invest 103:731-738 (1999).

Kawahara K, Nishikiori M, Imai K, Kishi K, Fujiki Y: Radiographic observations of pyknodysostosis. Report of a case. Oral Surg Oral Med Oral Pathol 44:476-482 (1977).

-Khan B, Ahmed Z, Ahmad W: A novel missense mutation in cathepsin $\mathrm{K}$ (CTSK) gene in a consanguineous Pakistani family with pycnodysostosis. J Investig Med 58:720-724 (2010).

Kumar P, Henikoff S, Ng PC: Predicting the effects of coding non-synonymous variants on protein function using the SIFT algorithm. Nat Protoc 4:1073-1081 (2009).

Li HY, Ma HW, Wang HQ, Ma WH: Molecular analysis of a novel cathepsin K gene mutation in a Chinese child with pycnodysostosis. J Int Med Res 37:264-269 (2009).

- Li Z, Hou WS, Escalante-Torres CR, Gelb BD, Bromme D: Collagenase activity of cathepsin $\mathrm{K}$ depends on complex formation with chondroitin sulfate. J Biol Chem 277:2866928676 (2002).
Li Z, Kienetz M, Cherney MM, James MN, Brömme D: The crystal and molecular structures of a cathepsin K:chondroitin sulfate complex. J Mol Biol 383:78-91 (2008).

Michela D, Stefano R, Barbara T, Camillo R, Stefania A, et al: Molecular analysis and characterization of nine novel CTSK mutations in twelve patients affected by pycnodysostosis. Mutation in Brief \#961. Online. Hum Mutat 28:524-533 (2007)

Naeem M, Sheikh S, Ahmad W: A mutation in CTSK gene in an autosomal recessive pycnodysostosis family of Pakistani origin. BMC Med Genet 10:76 (2009).

Schilling AF, Mülhausen C, Lehmann W, Santer $\mathrm{R}$, Schinke T, et al: High bone mineral density in pycnodysostotic patients with a novel mutation in the propeptide of cathepsin $\mathrm{K}$. Osteoporos Int 18:659-669 (2007).

Sedano HD, Gorlin RJ, Anderson VE: Pycnodysostosis. Clinical and genetic considerations. Am J Dis Child116:70-77 (1968).

- Tezuka K, Tezuka Y, Maejima A, Sato T, Nemoto $\mathrm{K}$, et al: Molecular cloning of a possible cysteine proteinase predominantly expressed in osteoclasts. J Biol Chem 269:1106-1109 (1994).

Toral-López J, Gonzalez-Huerta LM, Sosa B, Orozco S, González HP, et al: Familial pycnodysostosis: identification of a novel mutation in the CTSK gene (cathepsin K). J Investig Med 59:277-280 (2011). 